Meta

Journal des traducteurs

Translators' Journal

\title{
La regulación legal de la traducción jurídica de documentos de la common law en España: el funcionalismo en términos de "universales", un estándar de equivalencia
}

\section{Elena Ferran Larraz}

Volume 55, numéro 2, juin 2010

URI : https://id.erudit.org/iderudit/044239ar

DOI : https://doi.org/10.7202/044239ar

Aller au sommaire du numéro

\section{Éditeur(s)}

Les Presses de l'Université de Montréal

\section{ISSN}

0026-0452 (imprimé)

1492-1421 (numérique)

\section{Découvrir la revue}

Citer cet article

Ferran Larraz, E. (2010). La regulación legal de la traducción jurídica de documentos de la common law en España: el funcionalismo en términos de “universales", un estándar de equivalencia. Meta, 55(2), 266-274.

https://doi.org/10.7202/044239ar
Résumé de l'article

Afin de déterminer quel est le concept de traduction nécessaire aux traducteurs en Espagne, nous nous proposons d'étudier tout d'abord la manière dont le droit espagnol envisage la traduction selon le contexte social international contemporain. En effet, ce n'est qu'après avoir examiné la loi que le concept de traduction juridique peut être établi. Puis, nous vérifierons, en appliquant le fonctionnalisme au domaine juridique, si la fonction principale du document, à savoir son efficacité juridique, est préservée. L'efficacité, sur le plan légal, du document juridique à traduire est le critère fondamental à respecter. Elle est définie dans le contexte d'une collaboration internationale dans le cadre de laquelle une loi ou un document étrangers doivent être reconnus ou appliqués selon les lois et les principes du droit international. La collaboration internationale est fondée sur la reconnaissance de bases communes (universaux jurilinguistiques) chez les pays concernés ainsi que sur le respect des différences. 


\title{
La regulación legal de la traducción jurídica de documentos de la common law en España: el funcionalismo en términos de "universales", un estándar de equivalencia
}

\author{
ELENA FERRAN LARRAZ \\ Universitat Rovira i Virgili, Tarragona, Espanya \\ elena.ferran@urv.cat
}

\begin{abstract}
RÉSUMÉ
Afin de déterminer quel est le concept de traduction nécessaire aux traducteurs en Espagne, nous nous proposons d'étudier tout d'abord la manière dont le droit espagnol envisage la traduction selon le contexte social international contemporain. En effet, ce n'est qu'après avoir examiné la loi que le concept de traduction juridique peut être établi. Puis, nous vérifierons, en appliquant le fonctionnalisme au domaine juridique, si la fonction principale du document, à savoir son efficacité juridique, est préservée. L'efficacité, sur le plan légal, du document juridique à traduire est le critère fondamental à respecter. Elle est définie dans le contexte d'une collaboration internationale dans le cadre de laquelle une loi ou un document étrangers doivent être reconnus ou appliqués selon les lois et les principes du droit international. La collaboration internationale est fondée sur la reconnaissance de bases communes (universaux jurilinguistiques) chez les pays concernés ainsi que sur le respect des différences.
\end{abstract}

\begin{abstract}
In order to determine which translation concept is needed by translators in Spain, we will examine how the concept of translation is envisaged by Spanish law in keeping with today's international sociological context. Only after examining the law can the legal translation concept be determined. Then, applying functionalism to the legal context, we will analyze whether the main function of the document, that is, its legal effectiveness, is preserved. The fundamental criterion required is the legal effectiveness of the legal document to be translated. Such effectiveness is defined in the context of an international collaboration in which foreign law or documents need to be recognized or implemented in a country according to the principles of international law. Such international collaboration is based on the acknowledgement of the common ground of the countries involved (jurilinguistic universals) and on respect for the differences.
\end{abstract}

\section{MOTS-CLÉS/KEYWORDS}

droit international privé, collaboration internationale, universaux juridiques, sociologie juridique, fonctionnalisme

international private law, international collaboration, legal universals, legal sociology, functionalism

\section{Introducción}

Como veremos, la noción que el propio derecho tiene de la traducción jurídica es funcionalista, pues está al servicio de la función de eficacia jurídica del documento que se produce en el contexto de la colaboración internacional. En consecuencia, 
creemos que se puede beneficiar de los estudios de los traductólogos funcionalistas que la conciben como acto de comunicación pragmática. Esa doble condición del objeto de este tipo de la traducción (el documento jurídico) de acto regulado por el derecho y acto sometido a las leyes de la comunicación, nos permite estudiar este tipo de traducción desde el derecho y desde la traductología, en ambos casos desde el funcionalismo en particular. En este caso la interdisciplinariedad se establece entre la ciencia jurídica y la traductología. Este enfoque ya ha sido adoptado por otros tratadistas en traducción jurídica como Terral (2002) y Sarcevic (1997).

La teoría sobre traducción jurídica puede verse beneficiada por el estudio y aplicación de las conclusiones de la teoría general de la traducción. De hecho, es bastante escasa y se produce de forma bastante concisa en sus comienzos, preferentemente en el ámbito de la terminología. Sólo recientemente se han dado estudios teóricos sobre la traducción jurídica, inspirándose sólo en parte en los estudios generales sobre traducción (Marín 1996; Borja Albi 2000; Alcaraz y Hughes 2002; Monzó 2002; Terral 2002; Mayoral 2003). Ello comporta la ventaja que supone una extrapolación de los resultados obtenidos en los estudios sobre traducción general, pero tiene el riesgo de no tener en cuenta suficientemente la verdadera naturaleza de la traducción jurídica. Por esta razón, nosotros preferimos ahondar en la naturaleza de este tipo muy especial de traducción en el apartado a continuación, desde la regulación legal y desde la práctica de la traducción jurídica, evitando en todo momento extrapolar mecánicamente los principios que se aplican a otros tipos de traducción. Realizamos el proceso inverso: sólo un estudio de traducción jurídica y del documento jurídico desde el derecho de forma independiente nos permite después acudir a la teoría general sobre traducción.

\section{La regulación legal y la práctica de la traducción jurídica en España}

\subsection{El derecho y la traducción jurídica}

El derecho español regula la traducción jurídica de forma muy escasa mediante la Orden de 8 de febrero de 1996, que establece que el traductor jurado debe mencionar al final del texto meta en castellano que la traducción es una traducción fiel y completa del original en inglés.

Existe en la ley una referencia a la fidelidad, pero subsiste la incógnita: ¿en qué consiste esa fidelidad? A falta de una regulación más detallada, nos remitimos al concepto de documento jurídico en tanto que objeto de la traducción jurídica: creemos que la naturaleza y finalidad del documento jurídico consiste en producir efectos jurídicos y la traducción jurídica queda definida como colaboradora con dicha eficacia jurídica.

Esa eficacia jurídica, además, en ocasiones, es extraterritorial, de forma que el documento extranjero en España (de la common law, por ejemplo) tiene la vocación de producir algunos o todos sus efectos fuera del país de otorgamiento. En virtud de la colaboración internacional entre los países, el país donde se producirán esos efectos se hace cargo de garantizarlos. Así, por ejemplo, ejecuta una sentencia extranjera; admite a trámite una demanda por daños cometidos por un extranjero en el territorio; formaliza notarialmente el testamento de un inglés, etc.

La función del texto meta es, por tanto, colaborar con la eficacia jurídica del texto origen. ¿Cómo colabora? No se limita a informar en abstracto sobre la eficacia jurídica 
de un documento, sino que informa con finalidades jurídicas diferentes: colaborar a la prueba en un juicio, colaborar a la formación del consentimiento del que firma un contrato que no entiende, colaborar con el juez que desea conocer el derecho extranjero que debe aplicar en un litigio sobre un documento extranjero.

Nótese que decimos colaborar, pues el texto meta no es el medio de prueba en sí mismo, no es una versión del contrato que se firma, sino su mera traducción. De hecho, en la mayor parte de los casos el texto meta no tiene eficacia jurídica propia, simplemente colabora con la eficacia jurídica del documento de partida, suministrando la información de su contenido en el contexto y para la finalidad de que se trate.

Sin embargo, en otros supuestos el texto meta tiene una doble función: no sólo tiene la función de informar sobre la eficacia jurídica del documento meta, sino también la de erigirse en otra versión jurídica eficaz del documento de partida. Es el caso de la ley que se promulga en varias versiones que se suelen predicar como igualmente válidas y eficaces. Es el caso de documentos privados como el contrato o el testamento, cuando el otorgante establece que el acto jurídico se otorga en una doble versión, en inglés y en castellano, versiones igualmente válidas de un único negocio jurídico.

Pero sea en un supuesto de encargo de traducción u otro, el caso es que la ley española no desarrolla el criterio fundamental de la fidelidad ni propone criterios subsidiarios como puedan ser la naturalidad o adaptación a la cultura que recibe el texto y por eso es útil recordar la esencia de este tipo de traducción, que se concibe como colaboración con la eficacia jurídica y considerar también la práctica profesional como guía de traducción adicional.

\subsection{La práctica de la traducción jurídica en España}

A falta de regulación más prolija, observemos la práctica de la traducción en un supuesto límite de colaboración internacional donde el contexto es sumamente híbrido. Se trata del otorgamiento real de un testamento common law por un inglés en España ante un notario español y traductor jurado español: una auténtica labor de mediación.

A partir del ejemplo trataremos la pugna o alternancia entre las dos culturas jurídicas en juego en el texto meta en aras de

a) una fidelidad entendida como equivalencia funcional o suficiente (equivalencia de efectos jurídicos);

b) una adaptación al destinatario de la traducción, que en este caso es múltiple: el notario, que no sabe inglés, leerá la versión española; el testador, que no sabe español, leerá la versión inglesa, como también los beneficiarios anglosajones del testamento, que quizá tampoco conozcan el castellano. Veremos cómo el traductor opta en el texto meta por alternar entre las dos culturas en un esfuerzo por complacer a ese destinatario múltiple e híbrido.

El ejemplo del testamento sirve como exponente clarísimo de la colaboración entre los países, pues el notario español se presta a formalizar el testamento common law de un nacional inglés con bienes en España, es decir, un documento extranjero. El traductor jurado (por encargo del notario) busca el terreno de lo común entre las dos culturas y respeta sus diferencias, como vemos en la traducción de la cláusula de 
residuo, que transcribimos a continuación. Debe tenerse en cuenta que la primera versión se hizo en castellano por el notario y que sólo después se tradujo al inglés mediante el traductor jurado, pues tanto el notario como el testador no conocían el idioma del otro.

Nótese que la versión inglesa, el texto meta, es el resultado del encargo de traducción que el notario hace al traductor jurado. No sabemos si el notario, de palabra, dio al traductor instrucciones precisas sobre su traducción. Lo cierto es que aunque el notario no sabe inglés, valida el testamento como conjunto, en su doble versión y consecuentemente, la traducción realizada, de forma que el testamento se otorga en una doble versión. Hemos extraído la cláusula de residuo a modo de ejemplo, subrayando los culturemas por su interés traductológico.

Tras la cláusula de residuo a continuación (Tabla 1) incluimos una tabla sobre la estrategia utilizada para la solución de los culturemas y un comentario de ella (Tabla 2).

TABLA 1

\section{Muestra de traducción: la cláusula de residuo}

\begin{tabular}{|c|c|}
\hline Texto de partida & Texto meta \\
\hline $\begin{array}{l}\text { 1.- De todos sus bienes muebles e inmuebles, } \\
\text { derechos, valores, dinero, créditos, efectos y } \\
\text { demás, sean de la clase que fueren, existentes } \\
\text { en España, que le pertenezcan o posea el día } \\
\text { de su fallecimiento, instituye heredera a su } \\
\text { esposa: K. SCOTLAND. }\end{array}$ & $\begin{array}{l}\text { 1.- Of all his personal and real property, } \\
\text { rights, securities, monies, credits, effects and } \\
\text { other, whatever their kind, existing in Spain } \\
\text { which belong to him or which he may possess } \\
\text { on the day of his death, he institutes as his } \\
\text { heir his wife K. SCOTLAND. }\end{array}$ \\
\hline $\begin{array}{l}\text { Para el caso de que el instituido heredero } \\
\text { premuera al testador, no quiera o no pueda } \\
\text { heredarle, la sustituye por su hermano C. } \\
\text { SCOTLAND y RICH, Abogado, con } \\
\text { domicilio en la Calle _-_-_ no } 1 \text {, Surrey, Gran } \\
\text { Bretaña, como trustees nombrados por él, de } \\
\text { conformidad con su testamento otorgado en } \\
\text { Inglaterra y Gales, en concepto de trust para } \\
\text { sus hijos de su primer matrimonio y su } \\
\text { hijastra ELIZABETH en partes iguales. }\end{array}$ & $\begin{array}{l}\text { In the case that the appointed heir } \\
\text { predeceases the testator, does not want or is } \\
\text { unable to inherit him, he substitutes her by } \\
\text { his brother C SCOTLAND AND RICH, } \\
\text { (Solicitor) of n }{ }^{\circ} \text { 1_-___Road, Surrey, Great } \\
\text { B., as trustees appointed by him under his will } \\
\text { made in England and Wales, upon trust for } \\
\text { his children from his first marriage and his } \\
\text { step-child ELIZABETH in equal shares. }\end{array}$ \\
\hline
\end{tabular}

TABLA 2

Equivalencias extraídas de la cláusula de residuo y estrategia de traducción utilizada

\begin{tabular}{|l|l|l|}
\hline \multicolumn{1}{|c|}{ Texto de partida } & \multicolumn{1}{c|}{ Texto meta } & \multicolumn{1}{c|}{ Estrategia } \\
\hline $\begin{array}{l}\text { Bienes muebles e } \\
\text { inmuebles }\end{array}$ & Personal and real property & $\begin{array}{l}\text { Esta fórmula, tanto en una cultura } \\
\text { como en otra, tiene una voluntad } \\
\text { omnicomprensiva (todo tipo de } \\
\text { bienes) }\end{array}$ \\
\hline Trust & Trust & Fiel. Calco \\
\hline Instituye heredera & He institutes as his heir & Fiel, adaptado a la cultura española \\
\hline
\end{tabular}

En el ejemplo vemos cómo el traductor ha respetado el trust anglosajón, la división anglosajona de la propiedad en real property / personal property y el vocablo del lenguaje jurídico español instituyo herederos, adoptando, en este último caso, la fórmula autóctona con preferencia a la anglosajona, que se formula en términos de 
remanente: I hereby give, device and bequeath the residue to my children. El traductor favorece una u otra cultura siempre con respeto a los efectos jurídicos del documento. Es decir, conjuga el principio de fidelidad con el principio de adaptación, lo que conlleva la mayor adaptación al lector, si ello no perjudica la fidelidad. Así, optar por instituyo herederos / I institute as my heirs y no por dispongo del remanente / I hereby give, device and bequeath the remainder no sólo es fiel, sino que también se adapta más a la cultura española. Así, optar por personal and real property, no sólo es fiel, sino que también se adapta al lector anglosajón, que entiende mejor esa expresión que el calco habitual movable and immovable property. En este caso la fidelidad no exige el calco porque la intención jurídica de bienes muebles e inmuebles es la de abarcar todo tipo de bienes: se trata de la fórmula habitual para incluir todos los bienes del patrimonio del causante. En la common law la fórmula con una intención comunicativa equivalente es la de any personal or real property. Sin embargo, en el contexto de la hipoteca mobiliaria, se haría necesario el calco movable property, pues el traductor, en ese caso, tendría que evitar el salto hacia el concepto más amplio de personal property. La hipoteca mobiliaria, según el derecho español, sólo afecta a los muebles y no, con carácter general, a todos los derechos personales. Los derechos arrendaticios, por ejemplo, son derechos personales que no pueden ser objeto de hipoteca. El calco movable property sería, por tanto, más prudente que el salto personal property.

Tras analizar los ejemplos anteriores observamos y confirmamos los siguientes criterios:

a) Un criterio prioritario de fidelidad a los efectos jurídicos;

b) Un criterio subsidiario de adaptación a la cultura del lector y destinatario del texto meta que, en este caso, es múltiple e híbrido.

Concluimos que la noción que el propio derecho tiene de la traducción jurídica es funcionalista, pues reconoce la función social y de eficacia jurídica del documento en el contexto de la colaboración internacional y, según postulamos a continuación, se puede beneficiar de los estudios de los traductólogos funcionalistas, que la conciben como acto de comunicación pragmática.

\section{Teoría general de la traducción y traducción jurídica. El funcionalismo}

Con el auge de la lingüística textual, de los estudios culturales y del funcionalismo, ha cesado la supeditación de la traducción a la lingüística desde que se considera que el objeto de investigación de los estudios de traducción no son los elementos lingüísticos, sino el texto como un todo impregnado de una intención comunicativa y que se trata de encontrar un equivalente del texto y no de las palabras o de porciones de texto ( fidelidad). Este enfoque de la lingüística textual se relaciona directamente con la concepción funcionalista de la traducción.

El funcionalismo, cuando habla de la traducción general, baraja el concepto de texto meta equivalente al original y lo revisa a partir de los años 1970 para establecer que, en la práctica de la traducción, el texto meta en muchas ocasiones no es un equivalente del original, no tiene la vocación de serlo y quizá no es siempre deseable que lo sea. Por el contrario, el texto meta ha de cumplir la función comunicativa que le imprime el encargo de traducción, que, a su vez, entronca con el contexto o sistema 
cultural meta en que se produce la función y con los textos y convenciones de esa cultura jurídica (equivalente funcional). El texto meta tiene que funcionar en relación con los fines pretendidos por el cliente, la persona que encarga la traducción. La traducción tiene que serle útil a alguien, tiene que tener una finalidad. De ahí que se le denomine teoría del skopos o teoría de la finalidad (Nord 1991). En este sentido, Nord (1991: 26)define el skopos:

Translation is always realized for a target situation with its determining factors (recipient, time and place of reception, etc.), in which the target text is supposed to fulfill a certain function which can and, indeed, must be specified in advance.

Sin embargo, el lema de el texto meta al servicio de una situación comunicativa tiene un matiz importante en traducción jurídica. El funcionalismo de Nord (1991; 1997) es una teoría general que abarca todos los marcos culturales, pero el marco jurídico limita y especifica la teoría: se concreta y encuentra límites en las necesidades y garantías del derecho.

Según la propia teoría funcionalista sólo es posible que la función del texto meta difiera de la del texto original si varía la situación comunicativa en que se producen. Efectivamente, según Nord, la equivalencia se mide teniendo en cuenta la situación comunicativa en que se producen sendos mensajes, el texto origen y el texto meta: "Being culture-bound linguistic signs, both the source text and the target text are determined by the communicative situation in which they serve to convey a message" (Nord 1991: 7). Lo que ocurre es que en el caso de la traducción jurídica las situaciones comunicativas correspondientes a ambos textos son, en esencia, las mismas: tanto el texto origen como el texto meta se interpretan en términos de efectos jurídicos entendidos como universales suficientemente equivalentes.

Apliquemos el funcionalismo de Nord $(1991 ; 1997)$ al encargo de traducción jurídica que se produce en esa situación comunicativa internacional, normalmente entre dos especialistas. Según nuestra visión, la finalidad o skopos de la traducción jurídica que se imprime en el encargo de traducción es indefectiblemente conseguir que el texto origen surta los efectos jurídicos que le son propios. Se trata de un encargo estándar que viene determinado por la finalidad última del derecho: la producción de efectos jurídicos.

Nord también contempla este supuesto: "Functional equivalence between the source text and the target text is not the normal skopos of a translation, but an exceptional case in which the factor change of functions is assigned zero" (Nord 1991: 23).

La conclusión de este apartado es que la función del texto meta no varía en esencia de la del texto original. El funcionalismo establece que el texto meta debe ser el equivalente funcional. Para que se dé la equivalencia entre los textos, el traductor deberá conseguir un texto meta que refleje en esencia los efectos jurídicos deseados por el emisor. Para conseguir ese texto meta será necesaria una comparación entre los sistemas jurídicos en juego y la consiguiente transposición cultural, de forma que la equivalencia se presenta como aproximada, suficiente y esencial.

Utilizamos la expresión equivalente funcional en el primer sentido como equivalente funcional del texto como conjunto, sinónimo de texto meta suficientemente equivalente, como vemos a continuación, donde tratamos de la naturaleza cultural de la función. 


\section{Un texto suficientemente equivalente}

Según el funcionalismo, la función tiene siempre una naturaleza cultural que indica que es necesario que el traductor de un salto hacia la cultura jurídica receptora. También los tratadistas en traducción jurídica hablan en esos mismos términos (Schwab 1982; Gémar 1995; Marín 1996; Sarcevic 1997; Borja Albi 2000).

¿Es posible encontrar un equivalente funcional del texto en este supuesto, que supone una traslación de un ordenamiento jurídico al otro? En el caso de la traducción jurídica la conexión de un texto con el ordenamiento jurídico que lo regula es tan obligada y estrecha, que algunos dicen que es difícil o imposible encontrar un equivalente del texto origen. Borja (1998) se muestra más optimista y considera que la traducción jurídica es posible, aunque difícil, por las diferencias entre los ordenamientos jurídicos. Se expresa así respecto de la intraducibilidad o falta de equivalencia:

Estas dificultades, consecuencia directa de la gran carga sociocultural que tiene este tipo de traducción especializada, ha llevado a los autores a calificarla como una operación ínter lingüística y operación entre sistemas jurídicos, pues resulta imposible traducir toda la carga semántica de los textos legales sin establecer comparaciones jurídicas entre los sistemas de derecho de las respectivas lenguas. (Borja 1998: 101)

Sin embargo, una vez establecidas las diferencias culturales, es decir, las zonas de intraducibilidad, las zonas de riesgo, es tanto lo común entre las culturas que es posible una traducción en términos de eficacia jurídica, universal y común a los ordenamientos jurídicos, que se propone como aproximada. Esta es precisamente nuestra propuesta: la similitud o identidad de las necesidades humanas y su regulación por el derecho hace que exista un terreno común entre los ordenamientos jurídicos que hace posible la traducción, sin perjuicio de los supuestos de intraducibilidad.

Cuando hablamos de una equivalencia funcional, no se trata de preservar el texto original de forma absoluta, sino de encontrar aquél texto y aquéllas porciones de discurso que desempeñan una función jurídico-comunicativa equivalente o, mejor dicho, suficientemente equivalente en el ordenamiento jurídico de llegada. Así, el contract de la common law es suficientemente equivalente al contrato español. Igualmente, la cláusula de otorgamiento de un contrato español encuentra su correlato en una anglosajona aunque esta última tenga un redactado arcaico, como en el ejemplo a continuación:

(1) NOW, THEREFORE, for and in consideration of the sum of TEN DOLLARS (\$10.00), the covenants and obligations contained herein and other good and valuable consideration, the receipt and sufficiency of which is hereby acknowledged, the parties hereto hereby agree as follows: [...].

Sin embargo, esta transposición cultural no constituye una sumisión o inserción absoluta del documento en el sistema jurídico receptor, sino que se preserva la fidelidad a los efectos esenciales del documento. Así, siguiendo el ejemplo de la hipoteca anglosajona, en la traducción de un mortgage agreement típico el traductor necesariamente traducirá la cláusula traslativa de dominio a favor del acreedor hipotecario, rasgo esencial del derecho anglosajón. Igualmente, en la traducción del deed necesariamente indicará al lector español que se trata de un documento público y solemne que no puede equipararse a la escritura notarial por cuanto la cultura jurídica anglosajona no conoce la figura del notario de la civil law. Y ello a pesar de que muchas veces en la práctica de la traducción el deed se traduce como escritura. 


\section{Conclusiones}

La traducción jurídica está regulada por el derecho: no existe en la teoría sobre traducción jurídica una caracterización completa y explícita de la traducción jurídica, principalmente porque no existe una caracterización explícita del objeto de la traducción, a saber, el documento jurídico eficaz en tanto que regulado por el derecho y emitido en el contexto específico de la comunicación internacional. El documento jurídico eficaz es un objeto sui generis que prescribe y condiciona tanto el concepto como la práctica de la traducción jurídica. El aspecto jurídico del documento es determinante e ineludible para el traductor jurídico y debe regir su conducta de forma prioritaria, pues el traductor debe conseguir la equivalencia de los efectos jurídicos del documento de partida.

Concebimos un encargo estándar que pretende la equivalencia de efectos jurídicos del documento jurídico eficaz, de forma que la propia naturaleza del objeto de la traducción (el documento jurídico eficaz) comunica a toda la experiencia traductora ese ingrediente fundamental, estableciendo un criterio de fidelidad.

La fidelidad queda complementada por el criterio de adaptación: el consiguiente modelo de traducción postula la traducción de la eficacia jurídica del documento (principio de fidelidad), sin perjuicio de que el documento meta deba adaptarse al medio social que lo recibe (principio de adaptación). El principio de fidelidad es prioritario, de forma que se trata de preservar la fidelidad y, sólo subsidiariamente, de alcanzar la máxima adaptación a las convenciones de género de la cultura receptora.

El funcionalismo de Christiane Nord conforma nuestro modelo de traducción jurídica: además, la traductología y, más específicamente el funcionalismo de Christiane Nord, nos ha permitido desarrollar los rasgos de este modelo de traducción. De esta forma hablamos del equivalente funcional del texto como conjunto, de una equivalencia que se concibe como suficiente y de universales de eficacia jurídica (funciones jurilingüísticas) que sirven a este parámetro de la suficiencia, siempre con un reconocimiento de los intraducibles.

\section{REFERENCIAS}

Alcaraz Varó, Enrique y Hughes, Brian (2002): Legal Translation Explained. Manchester: St Jerome.

Bhatia, Vijay, Candlin, Chris y Engberg, Jan (2008): Legal Discourse across Cultures and Systems. Hong Kong: Hong Kong University Press.

BorJa AlBI, Anabel (1998): Estudio descriptivo de la traducción jurídica: un enfoque discursivo. Tesis doctoral. Barcelona: Universitat Autònoma de Barcelona.

Borja Albi, Anabel (2000): El texto jurídico inglés y su traducción al español. Barcelona: Ariel. GÉmAR, Jean-Claude (1995): Traduire ou l'art d'interpréter. Québec: Presses de l'Université du Québec.

Marín, Teresa (1996): La traducción de textos jurídicos ingleses. Tesis doctoral. Granada: Universidad de Granada.

Mayoral, Roberto (2003): Translating Official Documents. Manchester: St. Jerome.

Monzó, Ester (2002): La professió del traductor juridic i jurat: descripció sociologica del professional $i$ anàlisi discursiva del transgènere. Tesis doctoral. Castelló: Universitad Jaume I.

NorD, Christiane (1991): Text Analysis in Translation. Amsterdam: Rodopi.

Nord, Christiane (1997): Translating as a Purposeful Activity. Functionalist Approaches Explained. Manchester: St. Jerome. 
274 MeTA, LV, 2, 2010

SARCEvic, Susan (1997): A New Approach to Legal Translation. La Haya: Kluwer Law International.

Schwab, Wallace (1982): Fiducie en droit québécois en Langage du droit et traduction essais de jurilinguistique. In: Jean-Claude GÉmAR, ed. Langage du droit et traduction. The Language of the Law and Translation. Essais de jurilinguistique. Essays on Jurilinguistics. Montreal: Linguatech / Conseil de la langue française.

Terral, Florence (2002): La traduction juridique dans un contexte de pluralisme linguistique. Le cas du Règlement (CE) 40/94 sur la marque communautaire. Tesis doctoral. Barcelona: Universidad Autónoma de Barcelona. 